\title{
Magnetic Sensor Calibration and Residual Dipole Characterization for Application to Nanosatellites
}

\author{
John C. Springmann* and James W. Cutler ${ }^{\dagger}$ \\ University of Michigan, Ann Arbor, MI, 48109, USA \\ Hasan Bahcivan $\ddagger$ \\ SRI International, Menlo Park, CA, 94025, USA
}

\begin{abstract}
In this paper, we describe the techniques used to calibrate the individual (non-integrated) magnetometers onboard the Radio Aurora Explorer (RAX) satellite and describe a technique to characterize the residual dipole of small satellites. Magnetometer calibration is a critical part of RAX's magnetic-based attitude determination system. Our technique uses vector knowledge of the local magnetic field, a three-axis Helmholtz cage, and a leastsquares regression. Using this calibration, we can also quantify magnetometer uncertainty. The satellite residual dipole, in addition to affecting the onboard magnetic measurements, can be a dominant disturbance torque for nanosatellites. We present a method to characterize the residual dipole of nanosatellites that uses redundant external magnetic measurements, a least-squares minimization, and does not require large magnetically clean facilities. These techniques are used on RAX but can be extended to other fields of study involving magnetic measurements.
\end{abstract}

\section{Introduction}

This paper discusses two important topics related to the attitude determination and control system of satellites: magnetometer calibration and characterization of a satellite residual dipole. The ultimate goals of this work are to accurately calibrate nanosatellite-based magnetometers and to develop a reliable method to characterize the residual dipole of nanosatellites without the need for magnetically clean facilities. This work is important because magnetometer calibration is a critical requirement for magnetic-based attitude determination systems and the residual dipole affects both the magnetometer measurements and the satellite disturbance torques.

The calibration and dipole characterization techniques presented in this paper have been developed for use on the Radio Aurora Explorer (RAX) nanosatellite and applied in a university lab setting, but the work is general enough to be applied to other nanosatellites and various magnetic applications in other fields of study such as geophysics or the localization of metal objects. CubeSats and other nanosatellites are often built by university students, and access to clean magnetic facilities is not readily available.

\section{I.A. RAX Background}

The Radio Aurora Explorer (RAX) is a National Science Foundation funded 3U CubeSat (approximately $10 \mathrm{~cm} \times 10 \mathrm{~cm} \times 30 \mathrm{~cm}$ and $3 \mathrm{~kg}$ ) developed to study field aligned plasma irregularities in the lower polar ionosphere. ${ }^{1,2}$ RAX is a joint project between SRI International and the University of Michigan. The spacecraft has been designed, built, and tested by students at Michigan and is scheduled for launch in late 2010. Attitude control is accomplished with a passive magnetic system, which uses permanent magnets to keep the satellite aligned with Earth's magnetic field and hysteresis material to dampen oscillations, and attitude determination is accomplished via magnetometers, sun sensors, and rate gyros.

*Graduate Student, Aerospace Engineering, 1320 Beal Avenue, AIAA Member.

${ }^{\dagger}$ Assistant Professor, Aerospace Engineering, 1320 Beal Avenue, AIAA Member.

${ }^{\ddagger}$ Research Physicist, Geospace Studies, Engineering \& Systems, 333 Ravenswood Avenue. 


\section{I.B. Motivation and Related Work}

\section{I.B.1. Magnetic Attitude Determination}

Magnetometer calibration is a critical requirement for magnetic attitude determination systems and science missions that utilize magnetic field measurements. Magnetic attitude determination uses magnetometers onboard the spacecraft to measure the local vector of a known magnetic field such as Earth's field in the case of low Earth orbiting satellites. The spacecraft position is used to determine the expected magnetic vector at that location using a model such as the International Geomagnetic Reference Field (IGRF) or the World Magnetic Model (WMM). Spacecraft attitude is then determined by comparing the measured and modeled vectors. Magnetic attitude determination systems are common on nanosatellites given the low power and mass requirements of magnetometers and the well-known models of Earth's field in typical nanosatellite orbits. Magnetometers alone can determine attitude in two axes. Another reference vector, such as that to the sun measured via sun sensors, is required for full three-axis determination. For accurate determination, accurate pre-flight sensor calibration must be completed.

Magnetometer calibration is not new; various techniques exist in the literature. The text by Ripka ${ }^{3}$ discusses two means of calibrating magnetometers: by using the Earth's field and by using calibration coils. Various types of calibration coils, including circular and square Helmholtz coil pairs, are discussed. Wertz's text $^{4}$ on spacecraft attitude determination and control outlines the procedure to calibrate with Helmholtz coils and discusses magnetometer biases, axis non-orthogonality, and crosstalk. Techniques have also been developed that do not require vector magnetic field knowledge, such as those in References 5-8.

Traditional techniques where the external magnetic field vector is known are the basis of this work. We present our preliminary calibration method that utilizes Helmholtz coils. Future work includes expanding the calibration to include methods where vector knowledge is not required.

\section{I.B.2. Residual Dipole and Disturbance Torques}

Typical satellite disturbance torques include gravity gradient, aerodynamic drag, and solar pressure. The interaction between a satellite residual dipole and Earth's magnetic field causes an additional disturbance torque on the satellite. For nanosatellites with certain configurations, the residual dipole is the dominant disturbance torques acting on the satellite. We provide a comparison between the residual dipole and gravity gradient disturbances later in this section. We use gravity gradient in our comparison because it is typically the dominant disturbance torque at the altitudes of interest.

Magnetic torques affect satellite attitude through deliberate design of active or passive magnetic control systems or through inherent magnetic dipoles of the satellite, referred to as the residual dipole. The residual dipole is caused by electric currents and magnetic material within the satellite. The time-varying nature of the currents makes the residual dipole difficult to calculate. For missions where this torque is of interest, it is often more practical to measure the dipole experimentally rather than attempt a complex analytical calculation.

There are only a small number of nanosatellite residual dipole strengths presented in the literature. Two that are given are for the Space Dart ${ }^{9}$ and PACE ${ }^{10}$ CubeSats. The Space Dart residual dipole, characterized at the magnetic test facility at NASA Goddard Spaceflight Center, has a strength of $9 \times 10^{-3} \mathrm{~A} \cdot \mathrm{m}^{2}$. The residual dipole of the PACE CubeSat is estimated to be $5 \times 10^{-4} \mathrm{~A} \cdot \mathrm{m}^{2}$. We use these two numbers to provide a reasonable range of residual dipole strengths and compare the disturbance torques caused by the residual dipole and gravity gradient for a generic $3 \mathrm{U}$ CubeSat.

Our comparison is based on a $650 \mathrm{~km}, 72^{\circ}$ inclination orbit (that of RAX). The magnetic field at the corresponding spacecraft positions varies in magnitude between approximately 20 and $47 \mu \mathrm{T}$, thus our analysis uses field strengths of 20,34 , and $47 \mu \mathrm{T}$. We varied the residual dipole between 0 and $0.01 \mathrm{~A} \cdot \mathrm{m}^{2}$ and assumed the worst-case alignment between the satellite and Earth's field, which happens when the satellite magnetic dipole and Earth's field are perpendicular and the corresponding torque is simply the product of the two values. For the gravity gradient calculations, we assumed an evenly distributed $3 \mathrm{~kg}$ satellite mass with physical dimensions of $10 \mathrm{~cm} \times 10 \mathrm{~cm} \times 30 \mathrm{~cm}$, and satellite attitude that creates the highest possible gravity gradient torque. The resulting torques are shown in figure 1 . We see that with a residual dipole strength of $0.0008 \mathrm{~A} \cdot \mathrm{m}^{2}$, the worst-case residual dipole torque in a $47 \mu \mathrm{T}$ field becomes greater than the worst-case gravity gradient torque for the generic $3 \mathrm{U}$ CubeSat. In a field of $20 \mu \mathrm{T}$, the residual dipole torque is greater than gravity gradient torques if the residual dipole is greater than $0.0017 \mathrm{~A} \cdot \mathrm{m}^{2}$. This comparison 
confirms that the residual dipole is a dominant disturbance torque and its characterization is critical for an accurate disturbance force analysis.

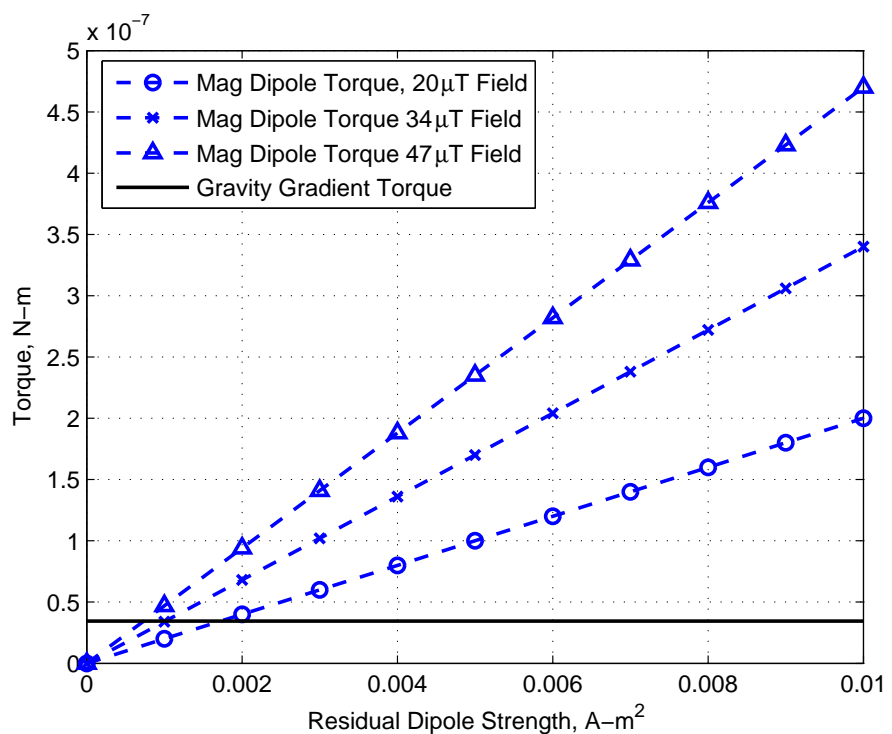

Figure 1. Worst-case residual dipole torque $(\mathrm{N} \cdot \mathrm{m})$ as a function of residual dipole strength (A $\left.\mathrm{m}^{2}\right)$ in fields of 20 , 34, and $47 \mu \mathrm{T}$ compared to the worst-case gravity gradient torque for a $3 \mathrm{U}$ CubeSat of generic mass properties and a 650 km orbit.

Work on characterizing satellite magnetic fields and the residual dipole dates back to the 1960s. Much work has been done at the NASA Goddard Spaceflight Center Magnetic Test Facility, including characterization of satellite magnetic fields using multi-pole expansion and determination of a corresponding dipole moment model. ${ }^{11-15}$ The facility is capable of cancelling Earth's magnetic field to a level of less than 0.5 $\mathrm{nT}^{13}$ which provides an excellent environment for such work.

Dipoles have also been characterized using a least-squares fitting method, which is the basis for this paper. Mehlem ${ }^{16}$ uses the technique to estimate multiple-dipole models of satellites and smaller pieces of hardware. $\mathrm{Hu}$ et $\mathrm{al}^{17}$ use a least-squares technique to track a magnetic object of known strength for medical purposes. The technique we present in this paper is a least squares minimization similar to the work of Mehlen and $\mathrm{Hu}$.

Magnetic dipole characterization has many applications besides spacecraft, such as geophysics applications and detection of metal objects. Much work has been done to characterize dipoles to various degrees of certainty for non-spacecraft applications. A very small sample of such techniques that are different than those used at NASA Goddard's facility include References 18-20.

The purpose of our residual dipole work is to develop a technique that doesn't require particularity clean facilities and that can be applied to nanosatellites and smaller pieces of hardware. We've shown the importance of this work for disturbance torque analysis, and the residual dipole work is also important in understanding the results of integrated magnetometer calibration because the satellite residual dipole will affect magnetometers onboard the spacecraft. Constant dipoles, such as those from magnetic material, will affect the magnetometers with a constant bias that resembles a hard-iron offset (a constant offset caused by the magnetic field from a nearby dipole). Time-varying dipoles can cause time-varying biases to affect the magnetometer.

The remainder of this paper is broken into two distinct sections. First, we discuss the procedures used for de-integrated magnetometer calibration for RAX and briefly discuss plans for integrated calibration. Second, we discuss the ongoing development and testing of the residual dipole characterization technique.

\section{Individual Magnetometer Calibration}

A three-axis Helmholtz cage ${ }^{21}$ is used to calibrate RAX's magnetometers. The cage can generate magnetic fields in three directions with strengths between approximately 0 and 140, -120 and 10, and -55 and $55 \mu \mathrm{T}$ in the cage $x, y$, and $z$ directions, respectively (this asymmetric range is governed by the heterogeneous 
power supplies for each axis and the ambient magnetic field at the location of the Helmholtz cage). The cage is used to calibrate all RAX magnetometers individually before integration and is also used to test the integrated magnetic attitude determination system.

To calibrate the individual magnetometers, we generate a series of static magnetic fields of various strengths, measure the fields with the magnetometer of interest, and use a linear least-squares fit to convert the sensor data to the true magnetic field strength. This section discusses the calibration of a PNI MicroMag3 three-axis magnetometer, one of six magnetometers in the RAX satellite. We use this same technique for all RAX magnetometers. Integrated magnetometer calibration is discussed in Section II.D, but the specifics are not presented because of delays in the RAX schedule.

\section{II.A. Calibration Technique and Results}

We use the Helmholtz cage to create a magnetic field profile of six static steps of various magnetic field strength as shown in figure 2. The control and knowledge of this profile is the critical component of the magnetometer calibration. The magnetic field is measured with a Honeywell HMR2300 magnetometer and the six-step profile is created five times to verify repeatability. The data from the five profiles is superimposed in figure 2. The repeatability and uncertainty of the magnetic field profiles is discussed in Section II.B. We choose to use the HMR2300 in our calibration because its resolution of $6.7 \mathrm{nT}$ is significantly better than the $32.0 \mathrm{nT}$ resolution of the PNI.

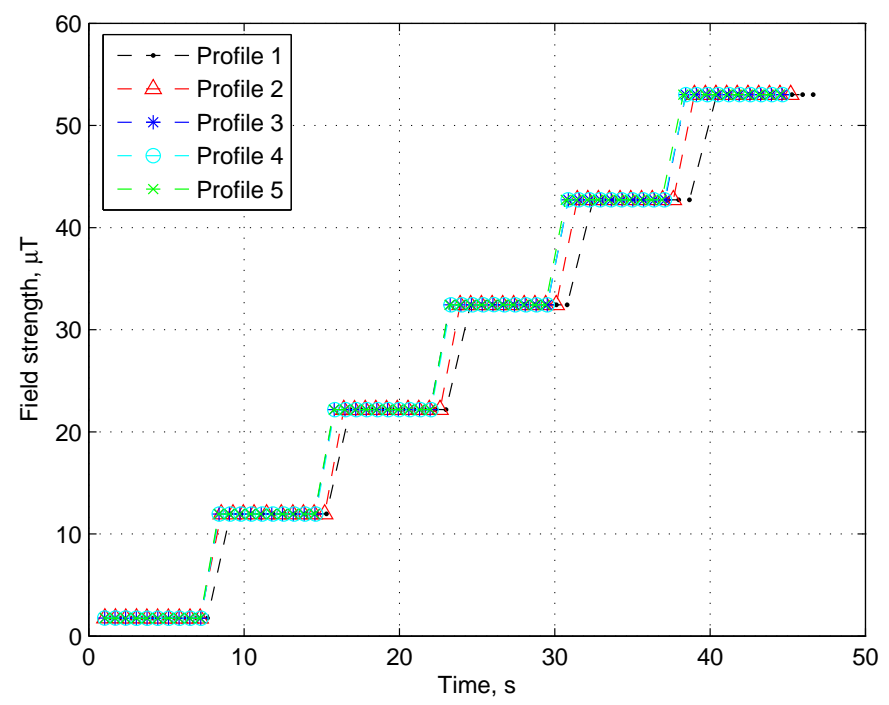

Figure 2. Truth data for a calibration sequence in the HMR2300 $x$-direction. The same profile is created in the $y$ and $z$ directions. There are six distinct magnetic field strengths created in increments of approximately $10 \mu \mathrm{T}$. The profiles are created five times consecutively and all five sets of data are superimposed in the plot

The mean value of the magnetic field at each step is assumed to be the true magnetic field strength for the corresponding Helmholtz cage power setting. We will refer to this data as the truth data. This highlights a potential weakness in this calibration technique: the calibration certainty is dictated by the certainty in the assumed known field strengths. Calibration uncertainty is discussed further in section II.B.

After the truth profiles are created and measured five times, the HMR2300 is replaced with the magnetometer to be calibrated - in this case, a PNI Micromag 3. (Five consecutive profiles was determined to be sufficient based on the standard deviation of the profile measurements.) We implement a linear least-squares regression to fit the PNI data to the truth data. The resulting calibration takes the form of Eq. (1), where $i$ is the axis, the calib and sensor subscripts indicate the calibrated and raw sensor data, respectively, and $m_{i}$ and $b_{i}$ represent the scaling factors and sensor offsets for each axis, respectively.

$$
P N I_{i, \text { calib }}=m_{i} \cdot P N I_{i, \text { sensor }}+b_{i}
$$

The scaling factor term, $m_{i}$, is a function of soft iron bias, axis non-orthogonality, and inherent scale factor errors. The offset term, $b_{i}$, is a function of hard iron bias and inherent sensor bias. 
In this calibration, the sensor and accompanying circuitry are de-integrated from the satellite. Magnetic biases and scaling factors from other satellite subsystems are not taken into account; magnetic effects from the integrated satellite will be quantified during integrated magnetometer testing.

The final calibration terms are the mean results of three trials. For the RAX PNI magnetometer, the calibration equations are given by Eqs. (2)-(4) and have units of $\mu \mathrm{T}$. Plots of the PNI data and to the truth data are shown in figures 3 and 4 .

$$
\begin{aligned}
& P N I_{x, \text { calib }}=1.0489 \cdot P N I_{x, \text { sensor }}-0.4987 \\
& P N I_{y, \text { calib }}=1.0706 \cdot P N I_{y, \text { sensor }}-0.3417 \\
& P N I_{z, \text { calib }}=0.8172 \cdot P N I_{z, \text { sensor }}-0.1261
\end{aligned}
$$

\section{II.B. Calibration Uncertainty}

There are various uncertainties associated with this type of calibration that must be quantified to determine the overall uncertainty of the magnetic measurements. The two main sources of uncertainty in the calibration are (1) uncertainty in the truth data (the basis of this calibration technique) and (2) alignment differences between the two magnetometers when the HMR2300 is replaced with the PNI.

We quantify uncertainties in the truth data with standard deviations of the HMR2300 measurements. There are two standard deviations of use: (1) the standard deviations of the measurements within a single step of each profile, and (2) the standard deviation of the mean strengths of the corresponding steps of each of the five profiles. The mean standard deviations of the measurements within each step are 4.1, 4.4, and 8.5 $\mathrm{nT}$ for the $x, y$, and $z$ directions of the $\mathrm{PNI}^{1}$ magnetometer, respectively. The standard deviations of the mean strength of each profile are 10.7, 7.5, and $26.4 \mathrm{nT}$, respectively. The standard deviation of the profiles is greater than the standard deviation of each individual step because of the discrepancies between the desired and actual voltage of the power supplies that drive the Helmholtz cage. Since the variation between the profiles is greater, we use the standard deviation between the profiles to quantify the uncertainty of the truth data. The uncertainty in the truth data is taken to be 10.7, 7.5, and $26.4 \mathrm{nT}$ for the $x, y$, and $z$ axes, respectively.

After the truth profiles are measured, the HMR2300 is replaced with the PNI. If there is a misalignment of angle $\theta$ between the magnetometers, the truth data will be incorrect by a factor of $(1-\cos (\theta))$. The magnetometers are placed by hand with drawn reference for alignment; no precise alignment instruments are used. Therefore, we quantify uncertainty with an estimated maximum misalignment of $\theta=5^{\circ}$ in each direction. The resulting uncertainty is a function of magnetic field strength rather than a constant offset.

The resulting total uncertainty in the calibration is given by Eqs. (5)-(7), where $B_{x, \text { truth }}, B_{y, \text { truth }}, B_{z, \text { truth }}$ are the truth magnetic field strengths $(\mu \mathrm{T})$ in each direction. By applying these equations to magnetic field strengths of $40 \mu \mathrm{T}$, we see that the uncertainties are $0.163,0.160$, and $0.179 \mu \mathrm{T}$ in each direction, or 0.41 , $0.40,0.45 \%$ of the true magnetic field strength, respectively.

$$
\begin{aligned}
& U_{x}=\left(1-\cos \left(5^{\circ}\right)\right) \cdot B_{x, \text { truth }}+0.0107 \\
& U_{y}=\left(1-\cos \left(5^{\circ}\right)\right) \cdot B_{y, \text { truth }}+0.0075 \\
& U_{z}=\left(1-\cos \left(5^{\circ}\right)\right) \cdot B_{z, \text { truth }}+0.0264
\end{aligned}
$$

\section{II.C. Magnetometer Uncertainty}

The previous section discussed certainty bounds of the magnetometer calibration. There is also uncertainty in the raw sensor measurements (before readings are converted with the calibration equations). This sensor uncertainty can be quantified with our magnetometer calibration technique. Standard deviations of the raw PNI data taken during exposure to the constant magnetic fields are computed. The standard deviation of the PNI data is significantly higher than that of the HMR data, which indicates that the standard deviation of the PNI is in fact an accurate representation of its uncertainty rather than a result of variation of the

\footnotetext{
${ }^{1}$ We reference the axes of the PNI because the axis orientation of the HMR and PNI was not the same due to their placement within the Helmholtz cage, but all results are presented in the PNI sensor frame.
} 


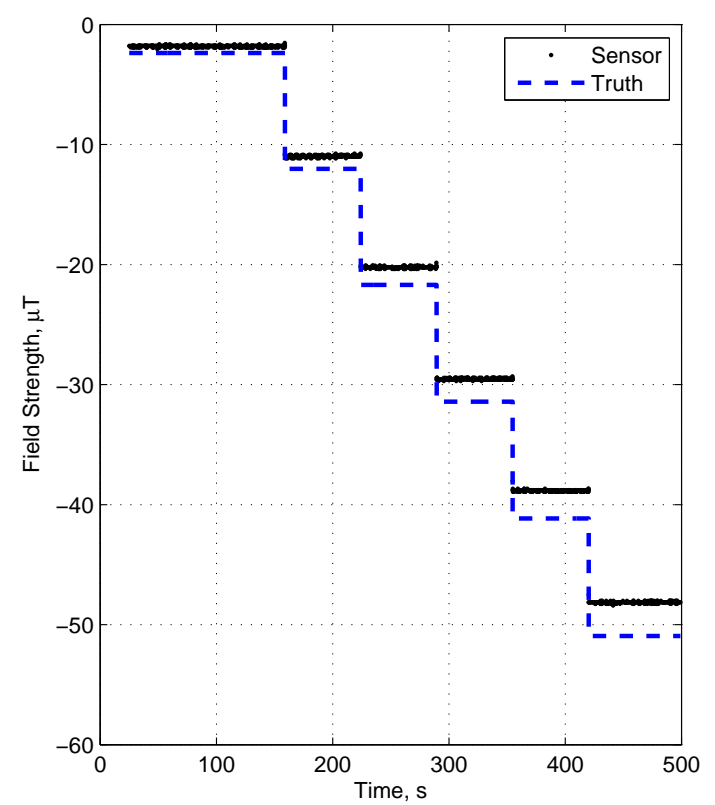

(a) PNI $x$-axis calibration data.

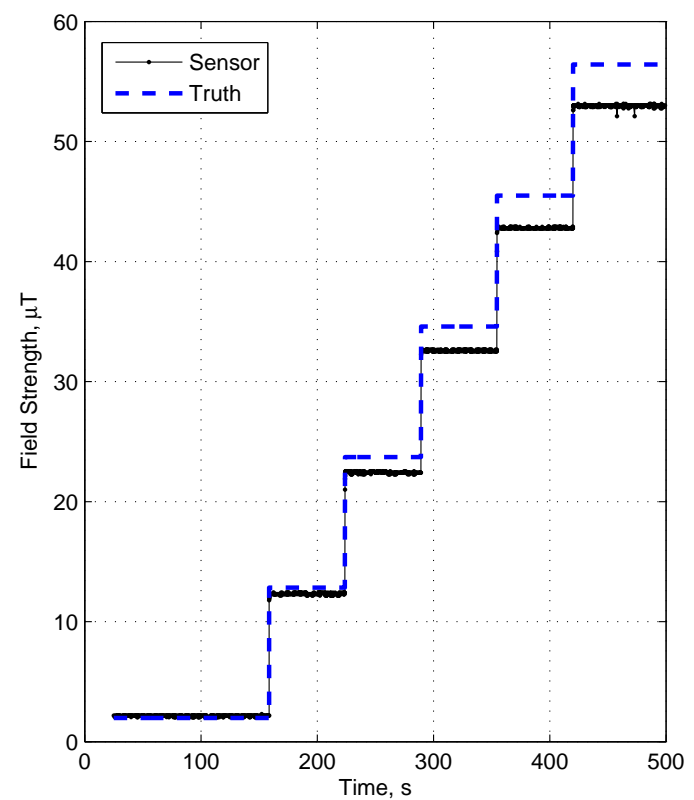

(b) PNI $y$-axis calibration data.

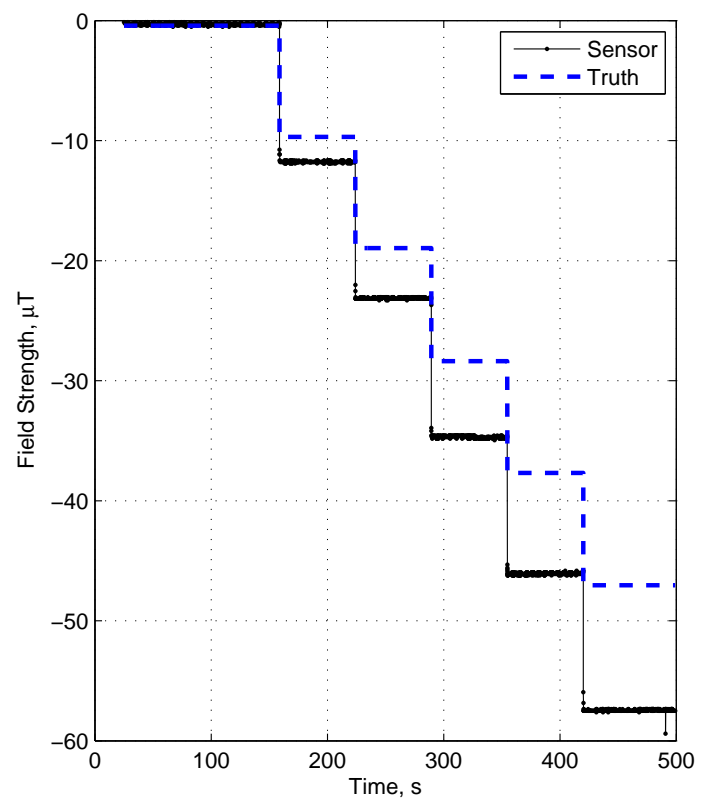

(c) PNI $z$-axis calibration data. The increasing gap between the sensor and truth data is represented in Eq. 4 by the 0.8172 scale factor.

Figure 3. Raw PNI data and truth data for each PNI axis. A least-squares regression is applied to this data to obtain the sensor calibration terms. 


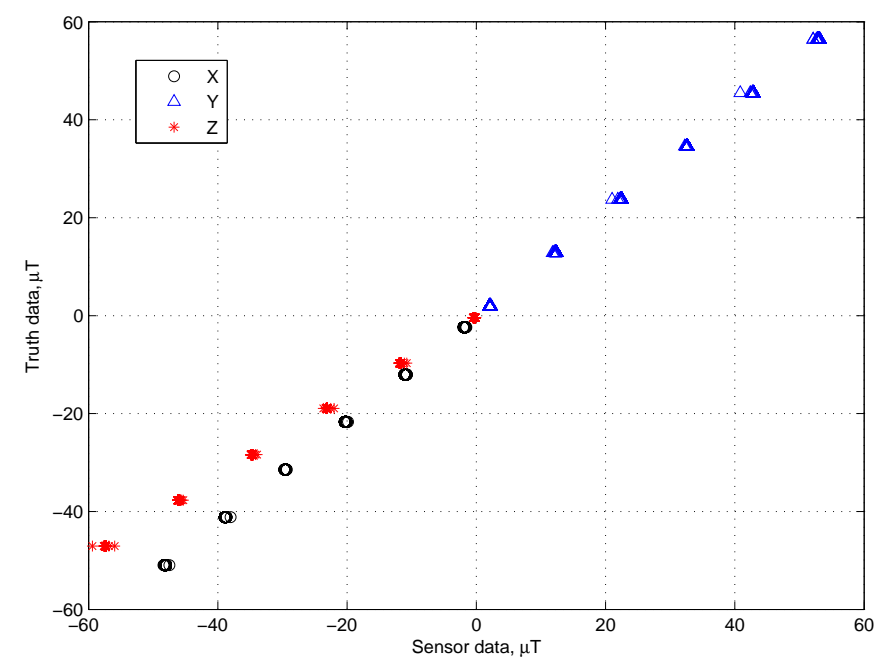

Figure 4. Truth data vs sensor data for all three axes. This is the same data as shown in figure 3 , but the PNI data are plotted as a function of truth data rather than time. The $x$ and $z$ axis data are negative because of the relative orientation between the axes of the PNI and Helmholtz cage. The fields used in the truth profile are either positive or negative rather than over a larger range because of limitations of the Helmholtz cage.

magnetic field. If the PNI standard deviations were equal to the HMR2300 standard deviations, we would not be able to distinguish between magnetometer uncertainty and variation in the magnetic fields generated by the Helmholtz cage.

To confirm the PNI standard deviations are a true indication of uncertainty, we also tested it inside a zero-gauss chamber. The zero-gauss chamber is a magnetically shielded chamber with a constant magnetic field of less than $300 \mathrm{nT}$ inside. To test uncertainty, we take at least 1000 data points inside the zero-gauss chamber and compute the standard deviation. The standard deviation is approximately the same value as that during the calibration within the Helmholtz cage, which confirms the uncertainty is that of the PNI rather than variation in the measured magnetic field.

The magnetometer uncertainty is defined to be the standard deviation from testing within the Helmholtz cage, and the resulting uncertainties are $0.072,0.081$, and $0.088 \mu \mathrm{T}$ in the $x, y$, and $z$ axes, respectively. The PNI MicroMag3 datasheet does not give certainty bounds, but the resolution of the magnetometer is 0.032 $\mu \mathrm{T} /$ count (counts are the output of the Analog-to-Digital Converter, i.e the raw sensor output). Thus the uncertainty we've measured experimentally corresponds to between 2 and 3 counts. This uncertainty is less than the uncertainty of the calibration which was discussed in Section II.B. The exact difference depends on the magnetic field strength.

\section{II.D. Integrated Magnetometer Testing}

In addition to de-integrated magnetometer calibration, the magnetometers must be calibrated when they are in the fully integrated satellite to quantify the magnetic effects of the integrated system. Satellite subsystems will induce hard-iron offsets and potentially scaling factors $\left(b_{i}\right.$, and $m_{i}$, respectively, from Eq. (1)). Integrated magnetometers will also experience satellite-induced noise that increases sensor uncertainty and decreases the accuracy of the magnetic attitude determination system. This increase in uncertainty must be quantified. (In addition to uncertainties of the magnetometer measurements, uncertainties of the reference magnetic field model, such as the IGRF or WMM, must be taken into account). We will use similar methods to those presented in Section II.A along with methods that do not require vector knowledge of the external magnetic field ${ }^{8}$ to calibrate the integrated magnetometers. The integrated calibration is not presented in this paper because of delays in the RAX testing schedule.

Many of the integrated satellite magnetic effects can be quantified by the spacecraft residual dipole. The residual dipole will affect the readings of the integrated magnetometers, and integrated magnetometer calibration, combined with a residual dipole characterization of the satellite, will provide a better understanding of the integrated magnetometer readings than calibration alone. And as discussed in Section I.B.2, 
the residual dipole causes a large disturbance torque. The next section of this paper describes preliminary work on satellite residual dipole determination using external magnetometers.

\section{Residual Dipole Determination}

We estimate the residual dipole of nanosatellites by fitting external magnetic field measurements to a single dipole model. We use a fitting algorithm to estimate the location, orientation, and strength of this dipole. The method is well-suited for nanosatellites because given their small size, it is possible to take many magnetic measurements around the satellite without the need for large fixtures.

\section{III.A. Background Equations and Method}

The magnetic field generated by a magnetic dipole in free space is given below by Eq. $(8),{ }^{22}$ where $\vec{B}(x, y, z)$ is the magnetic field at location $(x, y, z), \mu_{0}$ is the permeability of free space, $\vec{m}$ is the magnetic dipole moment (sometimes referred to as only magnetic moment or magnetic dipole), $\vec{r}$ is the vector from the dipole to $(x, y, z)$, and $\hat{r}$ is a unit vector in the direction of $\vec{r}$.

$$
\vec{B}(x, y, z)=\frac{\mu_{0}}{4 \pi}\left[\frac{3 \hat{r}(\hat{r} \cdot \vec{m})-\vec{m}}{|\vec{r}|^{3}}\right]
$$

After some manipulation, we obtain equations that describe the components of the magnetic field $\left(B_{x}, B_{y}, B_{z}\right)$ as a function of location $(x, y, z)$ due to a magnetic dipole located at $(a, b, c)$. The magnetic dipole has orientation described by a unit vector with components $(m, n, p)$ and a total dipole strength of $S$. The resulting equations are (9)-(11), where $R$ is the distance from the dipole to $(x, y, z)$ and is given by Eq. (12).

$$
\begin{gathered}
B_{x}=\frac{\mu_{0} \cdot S}{4 \pi}\left[\frac{3[(m(x-a)+n(y-b)+p(z-c)](x-a)}{R^{5}}-\frac{m}{R^{3}}\right] \\
B_{y}=\frac{\mu_{0} \cdot S}{4 \pi}\left[\frac{3[(m(x-a)+n(y-b)+p(z-c)](y-b)}{R^{5}}-\frac{n}{R^{3}}\right] \\
B_{z}=\frac{\mu_{0} \cdot S}{4 \pi}\left[\frac{3[(m(x-a)+n(y-b)+p(z-c)](z-c)}{R^{5}}-\frac{p}{R^{3}}\right] \\
R=\sqrt{(x-a)^{2}+(y-b)^{2}+(z-c)^{2}}
\end{gathered}
$$

Given magnetic field measurements at known locations, there are six unknowns in Eqs. (9)-(11). (There are actually 7 unknowns with the equations written as is, but the magnetic dipole orientation unit vector and strength can be combined to make three unknowns instead of four. That is, we can replace the unitvector constraint on orientation with a constraint that the magnitude is equal to $S$ and remove $S$ from the equations.) Because of sensor noise, many magnetic field measurements are taken and a non-linear leastsquares fitting algorithm is used to determine the dipole location, orientation, and strength from the external measurements. The function minimized is given in Eq. (13), where $i=1,2,3$ corresponds to the $x, y$, and $z$ axes, respectively, $B_{i}$ represents the thoretical magnetic field calculated from Eqs. (9)-(11), and $B_{i, \text { meas }}$ is the measured magnetic field strengths. We have used both ssqnonlin $^{23}$ and fmincon $^{24}$ functions in MATLAB to implement the minimization function and both have been found to work well.

$$
f(x)=\sum_{i=1}^{i=3}\left(B_{i}-B_{i, \text { meas }}\right) \cdot\left(B_{i}-B_{i, \text { meas }}\right)
$$

\section{III.B. Simulated Testing and Results}

We have tested the technique described in Section III.A with simulations to verify functionality, investigate performance, and to begin studying the dependence on the number of magnetometers and their locations. We present the results of six different numbers and locations of magnetometers, referred to as location schemes, around a $3 \mathrm{U}$ CubeSat volume $(10 \mathrm{~cm} \times 10 \mathrm{~cm} \times 30 \mathrm{~cm})$. The number and placement of the magnetometers around a $3 \mathrm{U}$ volume for each scheme is shown in figure 5 . 
To carry out the simulations, a dipole is simulated at location $(0,0,0)$ with an orientation unit vector of $(0,-1,0)$ and a strength of $0.05 \mathrm{~A} \cdot \mathrm{m}^{2}$. Analytical magnetic field values are calculated at each magnetometer location and random Gaussian noise is added to the measurements with standard deviations between 0 and $0.5 \mu \mathrm{T}$ in increments of $0.1 \mu \mathrm{T}$. The algorithm is then used to characterize the dipole from the noisy measurements.

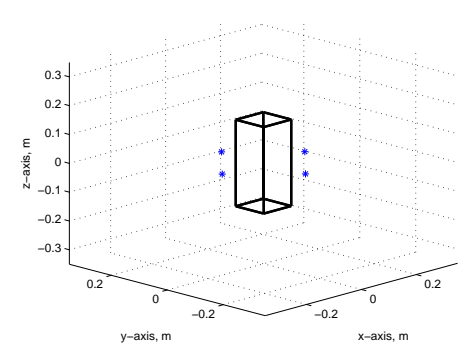

(a) Scheme 1. 4 magnetometers, each $5 \mathrm{~cm}$ away from the corresponding $\mathrm{x}$ or $y$ face of the $3 \mathrm{U}$ volume.

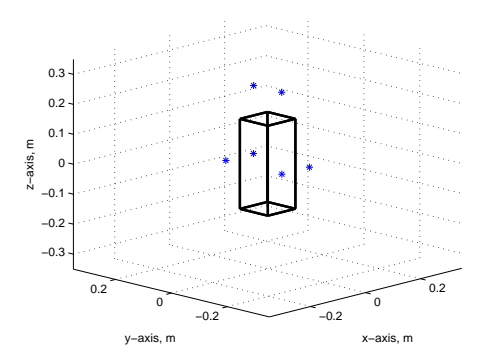

(c) Scheme 3. 6 magnetometers. 2 each off the $+y,-y$, and $+z$ face.

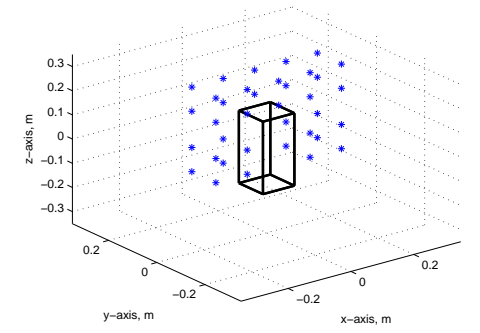

(e) Scheme 5. 36 magnetometers. 18 each parallel to the plane of the $+y$ and $-y$ surfaces.

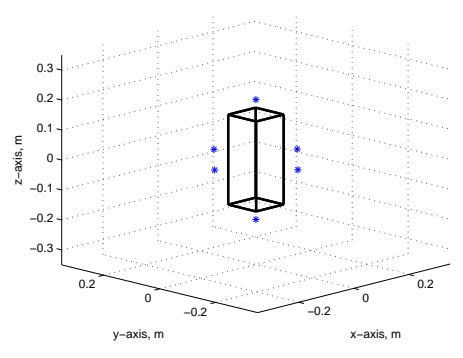

(b) Scheme 2. 6 magnetometers, each $5 \mathrm{~cm}$ away from the corresponding face of the $3 \mathrm{U}$ volume.

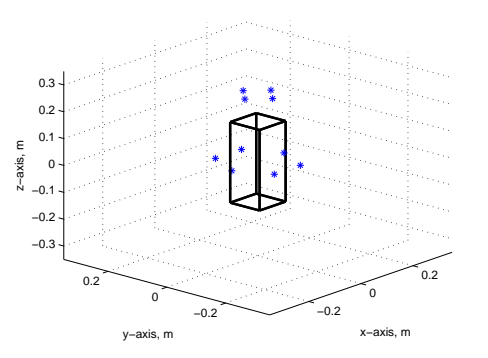

(d) Scheme 4. 10 magnetometers. Same as Scheme 3, but with additional magnetometers off the $+x$ and $-x$ faces as well as two more off the $+z$ face.

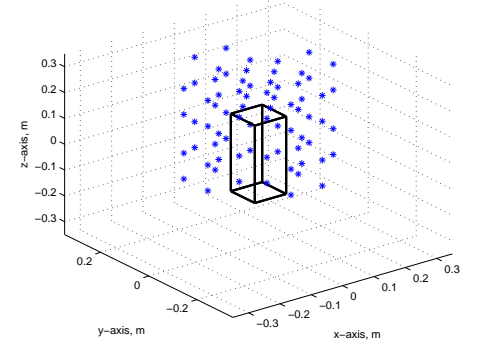

(f) Scheme 6. 72 magnetometers. Same as Scheme 5 but with two more planes parallel to the $+x$ and $-x$ surfaces.

Figure 5. Various external magnetometer location schemes. The enclosed volume in each plot is the size of a $3 U$ CubeSat.

Simulations were carried out for each magnetometer location scheme and noise level. The error in location, orientation, and strength between the resulting dipole and the simulated dipole is calculated for each location scheme and noise level. The errors of multiple trials are averaged and shown in figure 6 .

We see that the dipole is characterized perfectly if there is no sensor noise, verifying that the algorithm works, and the error in the dipole characterization increases as sensor noise increases. We see that location schemes 4-6 yield better results than schemes 1-3. This is expected because with noisy sensors, a larger number of measurements provides better results. We also see some dependence on magnetometer location. The worst results are from scheme 1, which has magnetometers in only one plane. Better results are seen 
with external magnetometers in multiple planes. Future work will include a more thorough study on the dependence on the number and location of the external magnetometers.
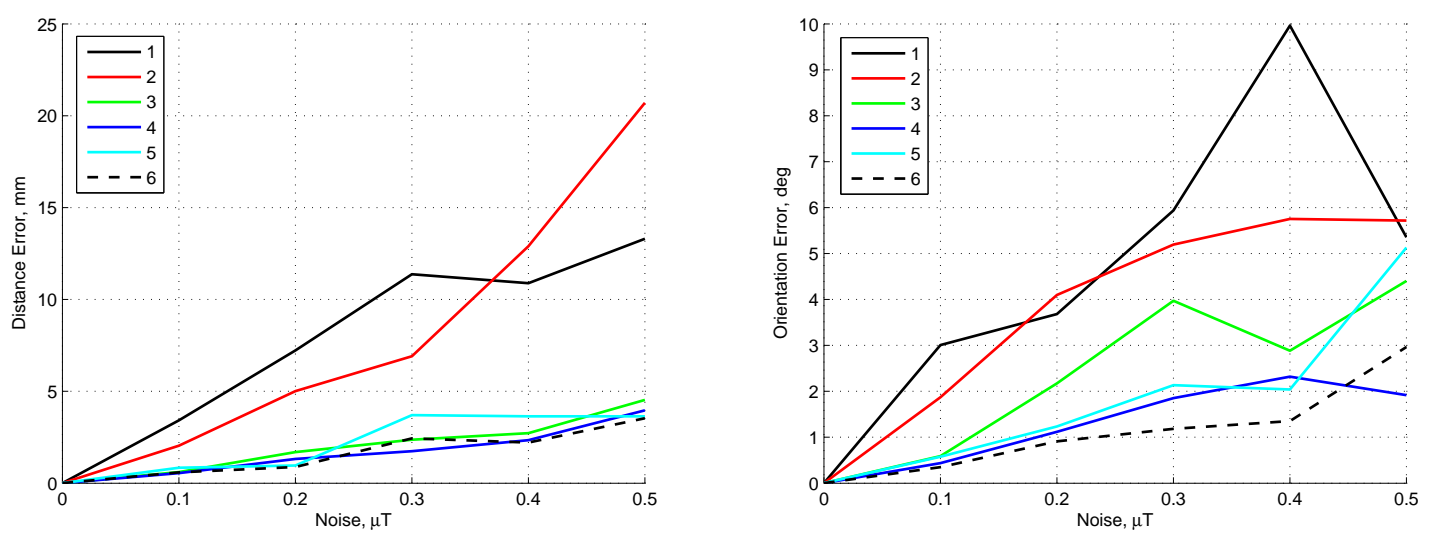

(a) Location error $(\mathrm{mm})$ as a function of sensor noise $(\mu \mathrm{T})$ (b) Orientation error $\left(^{\circ}\right)$ as a function of sensor noise $(\mu \mathrm{T})$

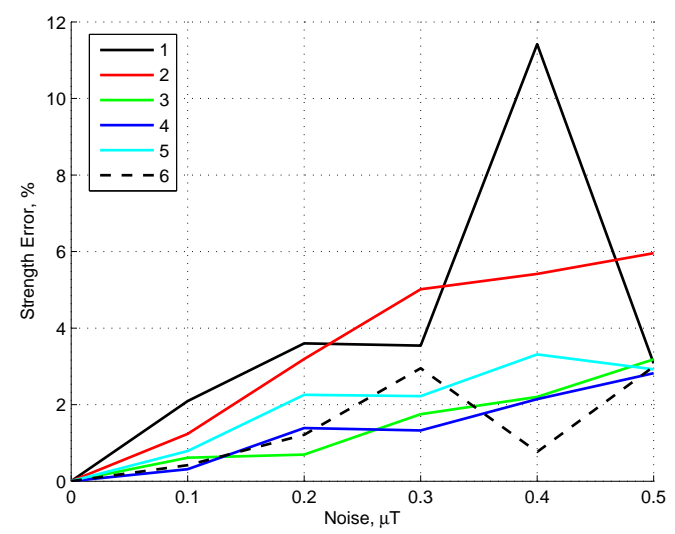

(c) Strength error $(\%)$ as a function of sensor noise $(\mu \mathrm{T})$

Figure 6. Errors between the estimated dipole parameters and the true dipole parameters. These are the average results from five trials at noise levels of $0,0.1,0.2,0.3,0.4$, and $0.5 \mu \mathrm{T}$ for each location scheme (1-6) shown in figure 5.

\section{III.C. Dependence on Initial Estimates}

Non-linear minimization functions have multiple solutions if there are multiple local minima in the region of interest. These functions require an initial estimate of the parameters as a starting point for the minimization. If there are multiple minima, the functions will typically converge to the minima that is closest to the initial parameter thus the algorithm will be dependent on the initial estimates. In this section, we look at the dependence of these initial parameters on the performance of the dipole estimation algorithms described in Section III.A. We study the affect of location, orientation, and strength, separately.

We use simulations in our initial study of parameter dependence and function behavior. We simulate a dipole at location $(0,0,0)$, an arbitrary orientation, and dipole strength of $0.05 \mathrm{~A}-\mathrm{m}^{2}$. We simulate a perfect magnetometer at a location of $(10,10,10) \mathrm{cm}$ by calculating the theoretical fields at this location. This magnetic field is referred to as $\left(B_{x, t r u t h}, B_{y, \text { truth }}, B_{z, \text { truth }}\right)$. We first study the dependence of dipole location by holding the dipole orientation and strength constant while varying the dipole location estimate. We calculate the field from the various dipole locations and refer to this field as $\left(B_{x, \text { est }}, B_{y, \text { est }}, B_{z, \text { est }}\right)$. The purpose is to look at the difference between estimated and actual field values over the region of interest. If the behavior is convex, meaning there is a single global minimum, then the algorithms will function independently of initial estimate of dipole parameters. 
Figure 7(a) shows the error in magnetic field readings as a function of location estimate, where error is defined by Eq. (14) given below. $i=1,2,3$ corresponds to the $x, y$, and $z$ axis, respectively. This is the same function as that being minimized in the dipole fitting algorithm, but since we're working with only a single simulated three-axis magnetometer, we are dealing with scalar quantities rather than vectors.

$$
\text { error }=\sum_{i=1}^{i=3}\left(B_{i, e s t}-B_{i, \text { truth }}\right)^{2}
$$

Figure 7(a) shows the error as a function of estimates of $-30 \leq a \leq 30 \mathrm{~cm}$ and $-30 \leq b \leq 30 \mathrm{~cm}$. Figure 7 (b) shows the error as a function of estimates of $-10 \leq a \leq 10 \mathrm{~cm}$ and $-10 \leq b \leq 10 \mathrm{~cm}$. Figure 7(b) is convex; there is only one minimum in the region. Figure 7 (a) has the same minimum with the region, but if the algorithm starts at the peak error location of $(10,10,10) \mathrm{cm}$, any direction will decrease the error. So if the minimization function starts moving the wrong direction, it may never find the true minimum.

To handle this potential problem, constraints are placed on lsqnonlin and fmincon to restrict the solution to the volume enclosed by the magnetometers. That is, if we're taking measurements around a CubeSat or a smaller piece of hardware, we will restrict the algorithm to search only within the volume enclosed by the magnetometers. This keeps the behavior convex over the entire region as in figure $7(\mathrm{~b})$ and prevents moving the wrong direction in a scenario like figure $7(\mathrm{a})$.

In the preceding discussion, we studied error as a function of $a$ and $b$, but not $c$. The convex behavior of the function is the same if plotting versus $c$ rather than $a$ or $b$. Similar simulations show that function dependence on dipole orientation alone and strength alone is also convex. In this simulated work, we varied either location, orientation, or strength while holding the other parameters constant. To complete the study of the dependence on initial conditions, we must determine if the function is globally convex. This is being pursued through analytical work beyond the scope of this paper.

\section{III.D. Experimental Testing and Results}

The algorithm was tested experimentally by taking magnetometer readings around a small electromagnet. The electromagnet used was simply an off-the-shelf cylindrical coil of wire. The height of the coil is $18.4 \mathrm{~cm}$ and the outer diameter is $20.6 \mathrm{~cm}$. The exact number of turns is unknown. The magnetometer used was a three-axis Honeywell HMR2300. Data was taken around the sides, above, and below the magnet while running $300 \mathrm{~mA}$ through the wire. The data was taken inside the zero-gauss chamber to avoid magnetic noise and biases from nearby objects. At each magnetometer location, data is taken with the electromagnet turned off before it is turned on, and the difference in the on and off readings is used to characterize the dipole. Because of the small size of the hardware, we can use small zero-gauss chambers and do not require large magnetically clean facilities.

The magnetometer locations are shown in figure 8 , where $(0,0,0)$ is the bottom center of the magnet. Four measurements were taken radially outward from the dipole approximately 4.1,6.7, 9.2, and $11.7 \mathrm{~cm}$ away from the coil and every 90 degrees around it. Four measurements were taken at arbitrary locations approximately $4.3 \mathrm{~cm}$ above the dipole, and five measurements were taken at arbitrary locations approximately $6.8 \mathrm{~cm}$ below the dipole. The fitting algorithm was applied with both lsqnonlin and fmincon, and the results were essentially identical. The dipole location was constrained in all directions to be between -10 and $10 \mathrm{~cm}$. The strength and orientation were unconstrained. The initial parameter estimate, along with the resulting estimated dipole parameters, are shown in Table 1.

We do not know the exact strength of the electromagnet because the exact number of turns is unknown. We can use the coil's location and orientation to confirm the results without knowledge of the strength. The center of the coil was located at $(0,0,9.2) \mathrm{mm}$ and it was oriented such that the dipole orientation should be vertical. The dipole location estimated from the algorithm was $(0.5,0.5,4.6) \mathrm{mm}$, which is only 4.7 $\mathrm{mm}$ from the center of the magnet. The orientation estimated with the fitting algorithm is $0.57^{\circ}$ and $0.72^{\circ}$ from vertical for the lsqnonlin and fmincon results, respectively. Because the location and orientation are confirmed to be accurate, the strength must be correct (strength is a scaling factor in the equations). This experiment shows that the dipole characterization technique can be applied to successfully determine dipole parameters from magnetic field measurements.

One way to quantify the error is to look at the difference between the magnetic field measurements and the theoretical field produced by the dipole with the resulting parameters. As expected, both lsqnonlin and 


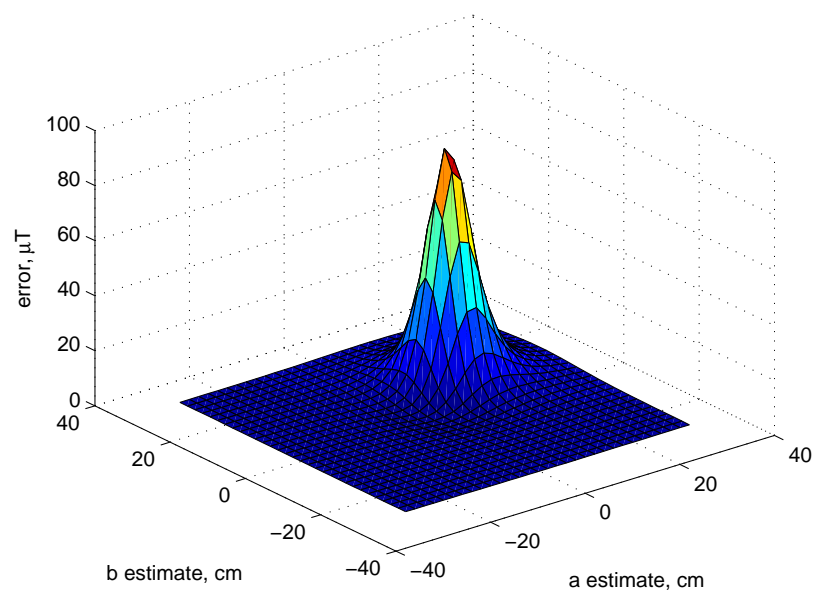

(a) $-30 \leq a \leq 30 \mathrm{~cm}$ and $-30 \leq b \leq 30 \mathrm{~cm}$

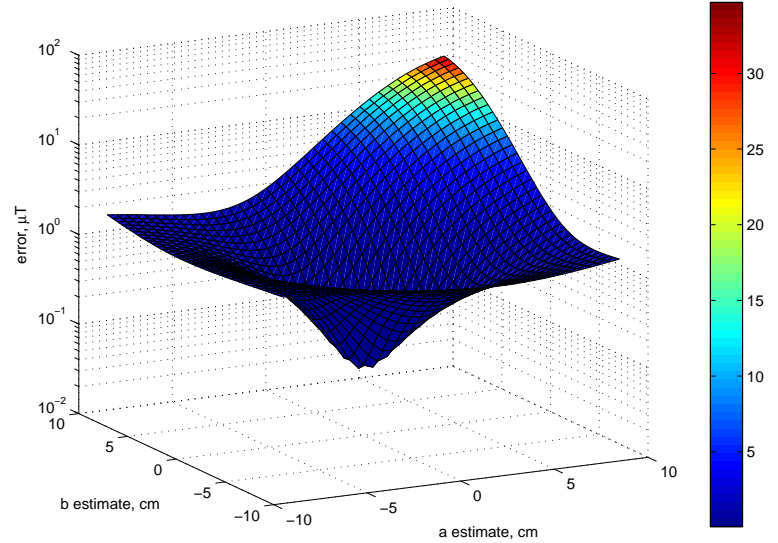

(b) $-10 \leq a \leq 10 \mathrm{~cm}$ and $-10 \leq b \leq 10 \mathrm{~cm}$ (log scale)

Figure 7. Magnetic field error $(\mu \mathrm{T})$ vs location estimate parameters $a$ and $b(\mathrm{~cm})$. The simulated magnetometer is located at $(10,10,10) \mathrm{cm}$.

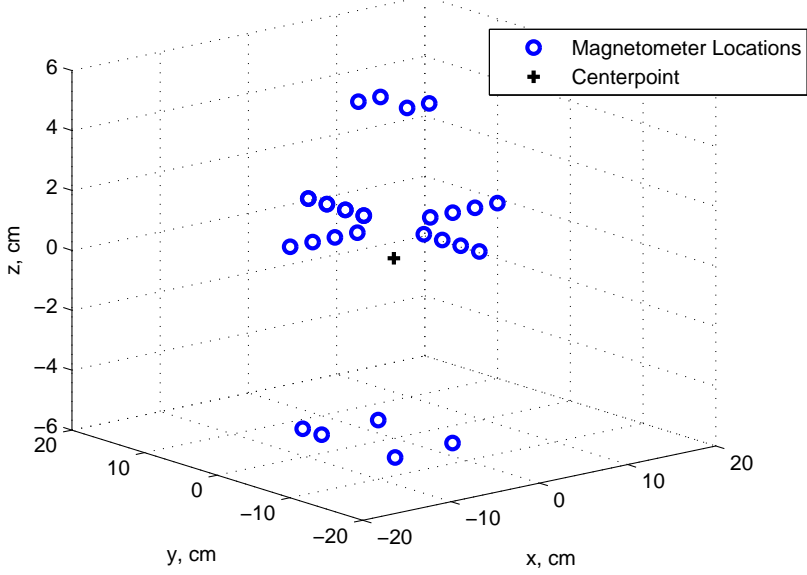

Figure 8. Magnetometer locations for experimental dipole characterization 
fmincon converged to the same difference. The difference is $-0.1784,0.1019$, and $-0.2108 \mu \mathrm{T}$ for the $x, y$, and $z$ field components, respectively.

Table 1. Experimental Dipole Characterization Parameters

\begin{tabular}{cccc}
\hline \hline Parameter & Algorithm Input & Parameters from lsqnonlin & Parameters from fmincon \\
\hline a (m) & 0 & 0.00048148 & 0.00048391 \\
b (m) & 0 & 0.0005007 & 0.00045824 \\
c (m) & 0 & 0.0046116 & 0.0046342 \\
m (-) & 0 & 0.0073957 & 0.007408 \\
n (-) & 0 & 0.0061363 & 0.010211 \\
p (-) & 1 & 0.99995 & 0.99992 \\
S (A.m $\left.{ }^{2}\right)$ & -0.0479 & -0.019326 & -0.019324 \\
\hline
\end{tabular}

\section{III.E. Residual Dipole Determination Future Plans}

The experimental dipole characterization work is still in its preliminary stages. Accuracy of the dipole estimation must be quantified. Through both simulation and theoretical development, the optimal location scheme and the required number of magnetometers around hardware of interest must be determined. The assumption of a single residual dipole model of the integrated satellite will be tested in future work. In addition to answering these questions, we will apply this technique to the RAX subsystems and the integrated RAX satellite. Application of the technique to RAX is not presented in this paper because of RAX schedule delays.

\section{Conclusion}

We have presented methods used for de-integrated magnetometer calibration for the RAX nanosatellite and preliminary work on residual dipole characterization. The magnetometer calibration uses a least-squares regression and relies on accurate vector knowledge of the local magnetic field, which we control with a threeaxis Helmholtz cage. The calibration improves the accuracy of the RAX attitude determination system, and the uncertainty analysis is a critical part of overall attitude determination accuracy.

Residual dipole characterization is important for magnetometer calibration because the spacecraft residual dipole will affect the onboard magnetic measurements. Additionally, the characterization is critical to quantify disturbance torques on nanosatellites. We've presented a method to characterize residual dipoles using multiple external measurements and a least-squares minimization technique. The experimental work can be done in a university-lab setting without the need for large magnetically clean facilities and accurate results have been demonstrated with both simulated and experimental work. This characterization is critical to understanding the disturbance forces acting on RAX in orbit and the active control requirements for future satellite missions. Both the magnetometer calibration and residual dipole characterization technique can easily be applied to non-satellite applications for use in other fields of work.

\section{Acknowledgements}

The authors are grateful to the RAX team members and the small satellite research group at the University of Michigan. This work is supported by NSF grant ATM-0838054.

\section{References}

\footnotetext{
${ }^{1}$ Moretto, T., "CubeSat Mission to Investigate Ionospheric Irregularities," Space Weather - The International Journal of Research and applications, Vol. 6, No. 11, Nov 12008.

${ }^{2}$ Bahcivan, H., Kelley, M. C., and Cutler, J. W., "Radar and rocket comparison of UHF radar scattering from auroral electrojet irregularities: Implications for a nanosatellite radar," Journal of Geophysical Research - Space Physics, Vol. 114, June 2009.

${ }^{3}$ Ripka, P., Magnetic Sensors and Magnetometers, Norwood, MA, 2001.

${ }^{4}$ Wertz, J., editor, Spacecraft attitude determination and control, Dordrecht, Holland, 1980.
} 
${ }^{5}$ Alonso, R. and Shuster, M. D., "Complete linear attitude-independent magnetometer calibration," Journal of the Astronautical Sciences, Vol. 50, No. 4, 2003, pp. $477-490$.

${ }^{6}$ Alonso, R. and Shuster, M. D., "TWOSTEP: A fast robust algorithm for attitude-independent magnetometer-bias determination," Journal of the Astronautical Sciences, Vol. 50, No. 4, 2003, pp. 433 - 451.

${ }^{7}$ Crassidis, J., Lai, K.-L., and Harman, R., "Real-time attitude-independent three-axis magnetometer calibration," Journal of Guidance, Control, and Dynamics, Vol. 28, No. 1, 2005, pp. $115-20$.

${ }^{8}$ Vasconcelos, J. F., Elkaim, G., Silvestre, C., Oliveira, P., , and Cardeira, B., "A Geometric Approach to Strapdown Magnetometer Calibration in Sensor Frame," FAC Workshop on Navigation, Guidance, and Control of Underwater Vehicles, Killaloe, Ireland, April 2008.

${ }^{9}$ Armstrong, J., Casey, C., Creamer, G., and Dutchover, G., "Pointing Control for Low Altitude Triple Cubesat Space Darts," 23rd Annual AIAA/USU Conference on Small Satellites, Logan, Utah, August 2009.

${ }^{10} \mathrm{Scholz}, \mathrm{A}$., Implementation of Advanced Attitude Determination and Control Techniques into a Nanosatellite, Master's thesis, National Cheng Kung University, Taiwan, 2007.

${ }^{11}$ Roy, T., "Spacecraft Magnetic-Field Modeling," IEEE Transactions on Magnetics, Vol. 13, No. 1, 1977, pp. $914-919$.

${ }^{12}$ Moskowitz, R. and Lynch, R., "Magnetostatic Measurement of Spacecraft Magnetic Dipole Moment," IEEE Transactions on Aerospace, Vol. AS 2, No. 2, 1964.

${ }^{13}$ Harris, P. K., "Near-Field Magnetic Dipole Moment Analysis." 2003, NASA Technical Report Document ID 20040016377.

${ }^{14}$ Neubauer, F. and Schatten, K., "Theoretical and observational analysis of spacecraft fields," Journal of Geophysical Research, Vol. 79, No. 10, 1974, pp. $1550-54$.

${ }^{15}$ Eichhorn, W. L., "Magnetic dipole moment determination by near-field analysis," NASA Technical Note D-6685, July 1972.

${ }^{16}$ Mehlem, K., "Multiple magnetic dipole modeling and field prediction of satellites," IEEE Transactions on Magnetics, Vol. MAG-14, No. 5, 1978, pp. $1064-71$.

${ }^{17} \mathrm{Hu}, \mathrm{C} .$, Meng, M. Q., and Mandal, M., "Efficient magnetic localization and orientation technique for capsule endoscopy," 2005 IEEE/RSJ International Conference on Intelligent Robots and Systems, Piscataway, NJ, USA, 2005, pp. 628 - 33.

${ }^{18} \mathrm{McFee}, \mathrm{J}$. and Das, Y., "Determination of the parameters of a dipole by measurement of its magnetic field [magnetometer sensors]," IEEE Transactions on Antennas and Propagation, Vol. AP-29, No. 2, March 1981, pp. $282-7$.

${ }^{19} \mathrm{McFee}$, J. and Das, Y., "Fast nonrecursive method for estimating location and dipole moment components of a static magnetic dipole," IEEE Transactions on Geoscience and Remote Sensing, Vol. GE-24, No. 5, Sept. 1986, pp. 663 - 73.

${ }^{20}$ Nara, T., Suzuki, S., and Ando, S., "A closed-form formula for magnetic dipole localization by measurement of its magnetic field and spatial gradients," IEEE Transactions on Magnetics, Vol. 42, No. 10, 2006, pp. 3291 - 3.

${ }^{21}$ Klesh, A., Seagraves, S., Bennett, M., Boone, D., Cutler, J., and Bahcivan, H., "Dynamically Driven Helmholtz Cage for Experimental Magnetic Attitude Determination," Vol. 135 of Advances in the Astronautical Sciences, 2009, In-print.

${ }^{22}$ Jackson, J., Classical electrodynamics, 2nd edition, Chichester, Sussex, UK, 1975.

${ }^{23}$ Mathworks, "lsqnonlin," http://www.mathworks.com/access/helpdesk/help/toolbox/optim/ug/lsqnonlin.html, Accessed June 2010.

${ }^{24}$ Mathworks, "fmincon," http://www.mathworks.fr/access/helpdesk/help/toolbox/optim/ug/fmincon.html, Accessed June 2010. 